\title{
Determination of Preferential Interaction Parameters by Multicomponent Diffusion. Applications to Poly(ethylene glycol)-Salt-Water Ternary Mixtures
}

\author{
Cong Tan, John G. Albright, and Onofrio Annunziata* \\ Department of Chemistry, Texas Christian University, Fort Worth, Texas 76129
}

Received: December 13, 2007; In Final Form: February 13, 2008

Poly(ethylene glycol) (PEG) is a hydrophilic nonionic polymer used in many biochemical and pharmaceutical applications. We report the four diffusion coefficients for the PEG- $\mathrm{KCl}-$ water ternary system at $25{ }^{\circ} \mathrm{C}$ using precision Rayleigh interferometry. Here, the molecular weight of PEG is $20 \mathrm{~kg} \mathrm{~mol}^{-1}$, which is comparable to that of proteins. The four diffusion coefficients are examined and used to determine thermodynamic preferential interaction coefficients. We find that the PEG preferential hydration in the presence of $\mathrm{KCl}$ is 1 order of magnitude larger than that previously obtained under the same conditions for lysozyme, a protein of similar molecular weight. In correspondence, the coupled diffusion in the PEG case was greater than that observed in the lysozyme case. We attribute this difference to the greater exposure of polymer coils to the surrounding fluid compared to that of globular compact proteins. Moreover, we observe that the PEG preferential hydration significantly decreases as salt concentration increases and attribute this behavior to the polymer collapse. Finally, we have also employed the equilibrium isopiestic method to validate the accuracy of the preferential interaction coefficients extracted from the diffusion coefficients. This experimental comparison represents an important contribution to the relation between diffusion and equilibrium thermodynamics.

\section{Introduction}

The chemical potential of macromolecules in aqueous solution is perturbed by the presence of osmolytes such as salts and small organic molecules. ${ }^{1-5}$ The primary thermodynamic effects of these additives on macromolecules are believed to be preferential solvation, ${ }^{1}$ binding, ${ }^{5}$ and Donnan equilibrium. ${ }^{6}$ Understanding how the chemical potential of a macromolecule is affected by the concentration and nature of osmolytes is important for characterizing free-energy changes of individual macromolecules and it is a necessary step for describing the collective behavior of macromolecules in solutions. By use of equilibrium dialysis ${ }^{7}$ and vapor pressure osmometry, ${ }^{8}$ preferential interaction coefficients have been determined for several protein-osmolyte aqueous solutions. ${ }^{3}$ These studies have been motivated by the strong interest in protein solubility, protein unfolding, and biochemical reactions. ${ }^{1-3}$

Macromolecule-osmolyte thermodynamic interactions are also central for understanding diffusive mass transport of macromolecules. ${ }^{9-13}$ Indeed a gradient of osmolyte concentration can generate a gradient of macromolecule chemical potential and vice versa. This is responsible for coupled diffusion in systems with two or more solutes. In the case of a macromolecule (1)-osmolyte (2)-solvent (0) ternary mixture, ${ }^{14}$ diffusion is described by the extended Fick's first law: ${ }^{15}$

$$
\begin{aligned}
& -J_{1}=D_{11} \nabla C_{1}+D_{12} \nabla C_{2} \\
& -J_{2}=D_{21} \nabla C_{1}+D_{22} \nabla C_{2}
\end{aligned}
$$

Here, $C_{1}$ and $C_{2}$ are molar concentrations of the two solutes, $J_{1}$ and $J_{2}$ are the corresponding molar fluxes, and the four $D_{i j}$ 's (with $i, j=1,2$ ) are the multicomponent diffusion coefficients.

* To whom correspondence should be addressed. E-mail: O.Annunziata@tcu.edu. Phone: (817) 257-6215. Fax: (817) 257-5851.
The main diffusion coefficients, $D_{11}$ and $D_{22}$, describe the flux of a solute due to its own concentration gradient, while crossdiffusion coefficients, $D_{12}$ and $D_{21}$, are responsible for the flux of a solute due to the concentration gradient of the other solute. We have previously shown that multicomponent diffusion can be used to extract preferential interaction coefficients for lysozyme-salt-water systems using precision Rayleigh interferometry. $6,10-13,16$

Here, we report multicomponent-diffusion measurements and preferential interactions coefficients for the poly(ethylene glycol) $-\mathrm{KCl}$-water ternary system at $25{ }^{\circ} \mathrm{C}$ using precision Rayleigh interferometry. In this work, the molecular weight of poly(ethylene glycol) (PEG) is $20 \mathrm{~kg} \mathrm{~mol}^{-1}$, comparable to that of proteins. This investigation has been motivated by the following three reasons. First, PEG is a hydrophilic nonionic polymer used in many applications including the partitioning and the precipitation of biomacromolecules using PEG-salt aqueous systems and the preparation of PEG-based materials for drug delivery applications. ${ }^{17-19}$ Knowledge of multicomponent diffusion coefficients and thermodynamic parameters on PEG-salt aqueous mixtures is generally important for the above applications. However, to our knowledge, measurements of preferential interaction coefficients for these systems have not been reported yet and diffusion coefficients for PEG-salt aqueous mixtures are only available for PEG oligomers. ${ }^{20,21}$ Second, preferential interaction coefficients have usually been determined for protein systems. A comparison between PEGsalt and protein-salt interactions will be valuable for understanding differences in hydration between PEG and proteins. Hence, we will compare our results with those previously obtained on the lysozyme- $\mathrm{KCl}$-water ternary system. ${ }^{6}$ Third, the determination of preferential interaction coefficients based on multicomponent diffusion assumes the validity of the three postulates of nonequilibrium thermodynamics. ${ }^{22}$ Thus an ex- 
perimental comparison between our diffusion-based method and equilibrium techniques represents a very important contribution to both equilibrium and nonequilibrium thermodynamics. We have made this comparison by measuring preferential interaction coefficients also using the isopiestic equilibrium method. ${ }^{23}$

\section{Theory}

We now outline the relationship between multicomponent diffusion and the preferential interaction coefficient. The diffusion coefficients in eq $1 \mathrm{a}, \mathrm{b}$ can be described relative to different reference frames: ${ }^{24}$ in the volume-fixed frame, the fluxes of the components of a ternary system satisfy $\left(J_{0}\right)_{\mathrm{v}} V_{0}+$ $\left(J_{1}\right)_{\mathrm{V}} V_{1}+\left(J_{2}\right)_{\mathrm{V}} V_{2}=0$; in the solvent-fixed frame, we have $\left(J_{0}\right)_{0}$ $=0$. Here, $J_{i}$ and $\bar{V}_{i}$ are the molar flux and partial molar volume of component $i$, respectively. The subscript "V" denotes the volume-fixed frame. The subscript " 0 " denotes the solvent component when appended directly to a flux and denotes the solvent-fixed frame when appended outside the parentheses to an already subscripted flux or diffusion coefficient. Since concentration differences are small and volume changes on mixing are negligible, our measurements correspond, to an excellent approximation, to the volume-fixed frame. ${ }^{24}$ Here, we report isothermal diffusion coefficients for both the volumeand solvent-fixed frames. The solvent-fixed frame coefficients, $\left(D_{i j}\right)_{0}$, are related to the volume-fixed frame coefficients $\left(D_{i j}\right)_{\mathrm{V}}$ by the following relations: ${ }^{25-27}$

$$
\begin{aligned}
& \left(D_{11}\right)_{0}= \\
& \left(D_{11}\right)_{\mathrm{V}}+\left[C_{1} /\left(1-C_{1} \bar{V}_{1}-C_{2} \bar{V}_{2}\right)\right]\left[\bar{V}_{1}\left(D_{11}\right)_{\mathrm{V}}+\bar{V}_{2}\left(D_{21}\right)_{\mathrm{V}}\right] \\
& \left(D_{12}\right)_{0}= \\
& \left(D_{12}\right)_{\mathrm{V}}+\left[C_{1} /\left(1-C_{1} \bar{V}_{1}-C_{2} \bar{V}_{2}\right)\right]\left[\bar{V}_{1}\left(D_{12}\right)_{\mathrm{V}}+\bar{V}_{2}\left(D_{22}\right)_{\mathrm{V}}\right] \\
& \left(D_{21}\right)_{0}= \\
& \left(D_{21}\right)_{\mathrm{V}}+\left[C_{2} /\left(1-C_{1} \bar{V}_{1}-C_{2} \bar{V}_{2}\right)\right]\left[\bar{V}_{1}\left(D_{11}\right)_{\mathrm{V}}+\bar{V}_{2}\left(D_{21}\right)_{\mathrm{V}}\right] \\
& \left(D_{22}\right)_{0}= \\
& \left(D_{22}\right)_{\mathrm{V}}+\left[C_{2} /\left(1-C_{1} \bar{V}_{1}-C_{2} \bar{V}_{2}\right)\right]\left[\bar{V}_{1}\left(D_{12}\right)_{\mathrm{V}}+\bar{V}_{2}\left(D_{22}\right)_{\mathrm{V}}\right]
\end{aligned}
$$

The linear laws of nonequilibrium thermodynamics for isothermal diffusion in terms of the Onsager diffusion coefficients (ODCs), $\left(L_{i j}\right)_{0}$, are simpler in the solvent-fixed frame. ${ }^{25-27}$ In this frame, the fluxes for a ternary system can be written as

$$
\begin{gathered}
-\left(J_{1}\right)_{0}=\left(D_{11}\right)_{0} \nabla C_{1}+\left(D_{12}\right)_{0} \nabla C_{2}=\left(L_{11}\right)_{0} \nabla \mu_{1}+ \\
\left(L_{12}\right)_{0} \nabla \mu_{2} \\
-\left(J_{2}\right)_{0}=\left(D_{21}\right)_{0} \nabla C_{1}+\left(D_{22}\right)_{0} \nabla C_{2}=\left(L_{21}\right)_{0} \nabla \mu_{1}+ \\
\left(L_{22}\right)_{0} \nabla \mu_{2}
\end{gathered}
$$

where $\mu_{i}$ is the chemical potential of the $i$ th component and

$$
\left(L_{12}\right)_{0}=\left(L_{21}\right)_{0}
$$

Equation 4 represents the Onsager Reciprocal Relation (ORR). ${ }^{28,29}$ The linear relations between fluxes and thermodynamic driving forces together with the ORR are the results of the three postulates of nonequilibrium thermodynamics. ${ }^{22}$ We can use eqs $3 \mathrm{a}$ and $3 \mathrm{~b}$ to relate the solvent-fixed diffusion coefficients and ODCs according to

$$
\begin{aligned}
& \left(D_{11}\right)_{0}=\left(L_{11}\right)_{0} u_{11}^{(c)}+\left(L_{12}\right)_{0} u_{21}^{(c)} \\
& \left(D_{12}\right)_{0}=\left(L_{11}\right)_{0} u_{12}^{(c)}+\left(L_{12}\right)_{0} \mu_{22}^{(c)}
\end{aligned}
$$

$$
\begin{aligned}
& \left(D_{21}\right)_{0}=\left(L_{21}\right)_{0} \mu_{11}^{(c)}+\left(L_{22}\right)_{0} \mu_{21}^{(c)} \\
& \left(D_{22}\right)_{0}=\left(L_{21}\right)_{0} \mu_{12}^{(c)}+\left(L_{22}\right)_{0} \mu_{22}^{(c)}
\end{aligned}
$$

where the $\mu_{i j}^{(c)}$ 's are defined by $\mu_{i j}^{(c)} \equiv\left(\partial \mu_{i} / \partial C_{j}\right)_{T, p, C k, k \neq j}$, where $T$ is the temperature and $p$ the pressure. ${ }^{25-29}$

The thermodynamics of macromolecule-salt aqueous mixtures is commonly described using molality-based chemical potential derivatives: $\mu_{i j}^{(m)} \equiv\left(\partial \mu_{i} / \partial m_{j}\right)_{T, p, m_{k}, k \neq j}$, where $\mu_{i}$ is the chemical potential of the $i$ th component and $\mu_{12}^{(m)}=\mu_{21}^{(m)}$. The $\mu_{i j}^{(m)}$, $\mathrm{s}$ are related to the $\mu_{i j}^{(c)}$, s by the following linear relations: ${ }^{29}$

$$
\begin{aligned}
&\left(C_{1} / m_{1}\right)\left(1-C_{1} \bar{V}_{1}-C_{2} \bar{V}_{2}\right) \mu_{11}^{(\mathrm{c})}= \\
&\left(1-C_{2} \bar{V}_{2}\right) \mu_{11}^{(m)}+C_{2} \bar{V}_{1} \mu_{12}^{(m)} \\
&\left(C_{1} / m_{1}\right)\left(1-C_{1} \bar{V}_{1}-C_{2} \bar{V}_{2}\right) \mu_{12}^{(\mathrm{c})}= \\
&\left(1-C_{1} \bar{V}_{1}\right) \mu_{12}^{(m)}+C_{1} \bar{V}_{2} \mu_{11}^{(m)} \\
&\left(C_{2} / m_{1}\right)\left(1-C_{1} \bar{V}_{1}-C_{2} \bar{V}_{2}\right) \mu_{21}^{(\mathrm{c})}= \\
&\left(1-C_{2} \bar{V}_{2}\right) \mu_{21}^{(m)}+C_{2} \bar{V}_{1} \mu_{22}^{(m)} \\
&\left(C_{2} / m_{1}\right)\left(1-C_{1} \bar{V}_{1}-C_{2} \bar{V}_{2}\right) \mu_{22}^{(\mathrm{c})}= \\
&\left(1-C_{1} \ddot{V}_{1}\right) \mu_{22}^{(m)}+C_{1} \bar{V}_{2} \mu_{21}^{(m)}
\end{aligned}
$$

The preferential interaction coefficient, $\Gamma_{12}$, is defined by: ${ }^{7,14}$

$$
\Gamma_{12} \equiv \lim _{m_{1} \rightarrow 0}\left(\frac{\partial m_{2}}{\partial m_{1}}\right)_{\mu 2, T, p}=-\lim _{m_{1} \rightarrow 0} \frac{\mu_{12}^{(m)}}{\mu_{22}^{(m)}}
$$

We now show how to relate $\Gamma_{12}$ to the solvent-frame diffusion coefficients. We can first solve eq $5 \mathrm{a}-\mathrm{d}$ with respect to $\left(L_{12}\right)_{0}$ and $\left(L_{21}\right)_{0}$. We can then use eq 4 to obtain:

$$
\left(D_{12}\right)_{0} \mu_{11}^{(c)}-\left(D_{11}\right)_{0} \mu_{12}^{(c)}=\left(D_{21}\right)_{0} \mu_{22}^{(c)}-\left(D_{22}\right)_{0} \mu_{21}^{(c)}
$$

By inserting eq $6 \mathrm{a}-\mathrm{d}$ into eq 8 and solving with respect to $\mu_{12}^{(m)} / \mu_{22}^{(m)}$, we obtain:

$$
\begin{aligned}
& \mu_{12}^{(m)} / \mu_{22}^{(m)}=\left\{\left(D_{21}\right)_{0}\left(1-C_{1} \bar{V}_{1}\right)-\left(D_{22}\right)_{0} C_{2} \bar{V}_{1}-\right. \\
& \left.\left(\mu_{11}^{(m)} / \mu_{22}^{(m)}\right)\left[\left(D_{12}\right)_{0}\left(1-C_{2} \bar{V}_{2}\right)-\left(D_{11}\right)_{0} C_{1} \bar{V}_{2}\right]\right\} /\left\{\left(D_{22}\right)_{0}(1-\right. \\
& \left.\left.C_{2} \bar{V}_{2}\right)-\left(D_{11}\right)_{0}\left(1-C_{1} \bar{V}_{1}\right)+\left(D_{12}\right)_{0} C_{2} \bar{V}_{1}-\left(D_{21}\right)_{0} C_{1} \bar{V}_{2}\right\}
\end{aligned}
$$

Equation 9 shows that ternary diffusion coefficients can be used to determine $\mu_{12}^{(m)} / \mu_{22}^{(m)}$, provided that $\mu_{11}^{(m)}$ and $\mu_{22}^{(m)}$ are known. We further remark that the accuracy of $\mu_{11}^{(m)}$ need not be high, i.e., $\approx 100 \%$ error in $\mu_{11}^{(m)}$ results in only $\approx 1 \%$ error in $\mu_{12}^{(m)} / \mu_{22}^{(m)}$. This implies that $\mu_{11}^{(m)}=1 / m_{1}$ is an excellent approximation for relatively low macromolecule concentrations $(\approx 10 \mathrm{mg} / \mathrm{mL})$. Indeed, several terms contribute only marginally to the value of $\mu_{12}^{(m)} / \mu_{22}^{(m)}$ and eq 9 can be approximately written as:

$$
\frac{\mu_{12}^{(m)}}{\mu_{22}^{(m)}} \approx \frac{\left(D_{21}\right)_{0}}{\left(D_{22}\right)_{0}}-C_{2} \bar{V}_{1}
$$

\section{Materials and Methods}

Materials. Poly(ethylene glycol) (PEG) with molecular weight ca. $20 \mathrm{~kg} \mathrm{~mol}^{-1}, 99 \%$ purity was purchased from Aldrich 
TABLE 1: Ternary Diffusion Coefficients for the PEG- $\mathrm{KCl}-\mathrm{H}_{2} \mathrm{O}$ System at $25{ }^{\circ} \mathrm{C}$

\begin{tabular}{llllllll}
\hline $\bar{C}_{1}(\mathrm{mM})$ & 0.2500 & 0.2500 & 0.2500 & 0.2500 & 0.2500 & 0.2500 & 0.2500 \\
$\bar{C}_{2}(\mathrm{M})$ & 0.2000 & 0.2500 & 0.3000 & 0.5000 & 0.9998 & 2.0000 & 2.2996 \\
$\bar{V}_{0}\left(\mathrm{M}^{-1}\right)$ & 0.01807 & 0.01807 & 0.01807 & 0.01806 & 0.01805 & 0.01801 & 0.01800 \\
$\bar{V}_{1}\left(\mathrm{M}^{-1}\right)$ & 16.76 & 16.77 & 16.76 & 16.79 & 16.84 & 16.92 & 16.95 \\
$\bar{V}_{2}\left(\mathrm{M}^{-1}\right)$ & 0.02819 & 0.02824 & 0.02836 & 0.02885 & 0.02991 & 0.03131 & 0.03164 \\
$\left(D_{11}\right)_{\mathrm{V}}\left(10^{-9} \mathrm{~m}^{2} \mathrm{~s}^{-1}\right)$ & $0.06010 \pm$ & $0.06016 \pm$ & $0.06082 \pm$ & $0.06107 \pm$ & $0.06230 \pm$ & $0.06356 \pm$ & $0.06393 \pm$ \\
& 0.00004 & 0.00007 & 0.00003 & 0.00012 & 0.00010 & 0.00016 & 0.00020 \\
$\left(D_{12}\right)_{\mathrm{V}}\left(10^{-9} \mathrm{~m}^{2} \mathrm{~s}^{-1}\right)$ & $0.000056 \pm$ & $0.000056 \pm$ & $0.000056 \pm$ & $0.000053 \pm$ & $0.000056 \pm$ & $0.000054 \pm$ & $0.000055 \pm$ \\
& 0.000001 & 0.000002 & 0.000001 & 0.000003 & 0.000002 & 0.000002 & 0.000003 \\
$\left(D_{21}\right)_{\mathrm{V}}\left(10^{-9} \mathrm{~m}^{2} \mathrm{~s}^{-1}\right)$ & $14.7 \pm 0.1$ & $17.4 \pm 0.2$ & $21.2 \pm 0.1$ & $32.2 \pm 0.3$ & $61.0 \pm 0.2$ & $119.0 \pm 0.9$ & $137.9 \pm 0.6$ \\
$\left(D_{22}\right)_{\mathrm{V}}\left(10^{-9} \mathrm{~m}^{2} \mathrm{~s}^{-1}\right)$ & $1.804 \pm 0.001$ & $1.809 \pm 0.002$ & $1.804 \pm 0.005$ & $1.816 \pm 0.002$ & $1.859 \pm 0.001$ & $1.963 \pm 0.002$ & $1.997 \pm 0.002$ \\
$\left(D_{11}\right)_{0}\left(10^{-9} \mathrm{~m}^{2} \mathrm{~s}^{-1}\right)$ & 0.06010 & 0.06048 & 0.06121 & 0.06160 & 0.06304 & 0.06489 & 0.06537 \\
$\left(D_{12}\right)_{0}\left(10^{-9} \mathrm{~m}^{2} \mathrm{~s}^{-1}\right)$ & 0.000070 & 0.000069 & 0.000069 & 0.000066 & 0.000070 & 0.000071 & 0.000072 \\
$\left(D_{21}\right)_{0}\left(10^{-9} \mathrm{~m}^{2} \mathrm{~s}^{-1}\right)$ & 14.7 & 17.8 & 21.6 & 33.2 & 64.0 & 129.3 & 151.5 \\
$\left(D_{22}\right)_{0}\left(10^{-9} \mathrm{~m}^{2} \mathrm{~s}^{-1}\right)$ & 1.804 & 1.822 & 1.820 & 1.843 & 1.919 & 2.097 & 2.162 \\
$\hat{\mu}_{11}^{(m)} / R T\left(\mathrm{Kg} \mathrm{mol}^{-1}\right)$ & 3949 & 3944 & 3938 & 3915 & 3857 & 3738 & 3697 \\
$\hat{\mu}_{22}^{(m)} / R T\left(\mathrm{Kg} \mathrm{mol}^{-1}\right)$ & 8.853 & 7.052 & 5.858 & 3.489 & 1.746 & 0.899 & 0.791 \\
$-\Gamma_{12}$ & $5.1 \pm 0.2$ & $5.7 \pm 0.2$ & $7.1 \pm 0.2$ & $10.0 \pm 0.3$ & $17.4 \pm 0.2$ & $30.2 \pm 0.6$ & $34.3 \pm 0.4$
\end{tabular}

and used without further purification. Because the polydispersity of PEG is given by a narrow Poisson distribution function, it does not significantly affect diffusion coefficients. Thus it is reasonable to assume that only one monodisperse macromolecule is present. ${ }^{30}$ The molecular weight, $M_{1}$, for PEG was taken to be $20000 \mathrm{~g} \mathrm{~mol}^{-1}$ and its density as $1.3 \mathrm{~g} \mathrm{~cm}^{-3}$ for buoyancy corrections. Deionized water was passed through a four-stage Millipore filter system to provide high purity water for all the experiments. The molecular weight of water, $M_{0}$, was taken as $18.015 \mathrm{~g} \mathrm{~cm}^{-3}$. Mallinckrodt AR $\mathrm{KCl}$ with $99.9 \%$ purity was dried by heating at $450{ }^{\circ} \mathrm{C}$ for $7 \mathrm{~h}$ and used without further purification. The molecular weight of $\mathrm{KCl}, \mathrm{M}_{2}$, was taken to be $74.55 \mathrm{~g} \mathrm{~mol}^{-1}$.

All solutions were prepared by mass with appropriate buoyancy corrections. Stock concentrated solutions of PEG were made by weight to an accuracy of $0.1 \mathrm{mg}$. Density measurements (Mettler-Paar DMA40 density meter) were made on the stock solutions for buoyancy corrections. The pairs of solutions for each diffusion experiment were prepared by weight. For binary PEG-water experiments, precise masses of PEG stock solutions were diluted with pure water to reach the final target PEG concentrations. For binary $\mathrm{KCl}$-water solutions, precise masses of pure salt were added to flasks and diluted with pure water to reach the final target $\mathrm{KCl}$ concentrations. For ternary $\mathrm{PEG}-$ $\mathrm{KCl}$-water solutions, precise masses of PEG stock solution and pure salt were added to flasks and diluted with pure water to reach the final target $\mathrm{PEG}$ and $\mathrm{KCl}$ concentrations. The densities of these solutions were measured to determine the corresponding molar concentrations and to calculate partial molar volumes.

Diffusion Measurements. Binary and ternary mutual diffusion coefficients were measured at $25.00{ }^{\circ} \mathrm{C}$ with the Gosting diffusiometer operating in the Rayleigh interferometric optical mode. ${ }^{10,11,31,32}$ The refractive index profile inside a diffusion cell is measured as described in ref 11 and references therein. We obtain 50 refractive index profiles during the course of each experiment. Experiments were performed by the free-diffusion method in a $10 \mathrm{~cm}$ vertical diffusion cell with a $2.5 \mathrm{~cm}$ horizontal optical path length and a $0.3 \mathrm{~cm}$ width. The temperature was regulated to $\pm 0.001{ }^{\circ} \mathrm{C}$ precision and $\pm 0.01{ }^{\circ} \mathrm{C}$ accuracy. Initial step-function distributions of solute concentrations were prepared with the boundary located at the center of the cell. All experimental data were obtained before detectable concentration changes occurred at the top and bottom ends of the cell, consistent with the free-diffusion boundary condition. A minimum of two experiments is required for determining the four diffusion coefficients at a given set of mean concentrations $\left(\bar{C}_{1}, \bar{C}_{2}\right.$ in Table 1$)$. These two experiments must have different combinations of solute concentration differences across the diffusion boundary. To verify reproducibility, two other duplicate experiments were performed at each set of mean concentrations.

Isopiestic Measurements. To verify the accuracy of the $\Gamma_{12}$ values obtained using eq 9 , we have also performed isopiestic measurements on the PEG-KCl-water ternary system. The isopiestic method is based on the chemical equilibrium of volatile components in a mixture. ${ }^{23}$ For the PEG (1) $-\mathrm{KCl}(2)-$ water (0) mixture, it is an excellent approximation to assume that water is the only component that is volatile. Mixtures of known weight and composition are placed inside open cups of inert metal (tantalum in our case), which are then placed in a copper block inside a desiccator. The desiccator is then partially evacuated and placed in a thermostatted water bath at $25.00 \pm$ $0.01{ }^{\circ} \mathrm{C}$. The solutions are allowed to reach equilibrium as the solvent redistributes itself among the mixtures. This equilibration process normally takes a time ranging from 1 to 4 weeks. After equilibrium has been reached, the chemical potential of the solvent is equal for all mixtures inside the desiccator. Note that isopiestic measurements are significantly longer than single diffusion measurements, which, normally, take less than $6 \mathrm{~h}$.

For each experiment, a given binary $\mathrm{KCl}$-water solution with known composition was prepared by weight and split into two solutions. A known amount of solid PEG was added to one of them. Four amounts of binary $\mathrm{KCl}$-water solutions and four amounts of ternary $\mathrm{PEG}-\mathrm{KCl}-$ water solutions were placed inside eight tantalum cups. The weights of the eight solutions were measured by first measuring the mass of each empty cup. Approximately $1 \mathrm{~g}$ of solution was placed in each cup and a preweighed cap with an O-ring seal was immediately placed on the cup. The assembled cap and the solution were weighed to $0.1 \mathrm{mg}$ precision, and the mass of solution calculated. The caps were removed and the cups were placed in the apparatus and equilibrated. When the chambers were open to remove the cups, the set of eight caps were simultaneously pushed onto the cups using a special cap holder. The cups were then lifted out of the copper block and weighed to $0.1 \mathrm{mg}$ precision. The final molalities were calculated from weight changes and initial concentrations.

For the four binary $\mathrm{KCl}$-water mixtures, the average $\mathrm{KCl}$ molality, $m_{2}^{\prime}$, was determined. For the four ternary PEG-KClwater solutions, the average PEG molality, $m_{1}$, and the average $\mathrm{KCl}$ molality, $m_{2}$, were determined. We have then computed the quantity $\left(m_{2}-m_{2}^{\prime}\right) / m_{1}$. Measurements at several values of $m_{1}$ allow us to determine $\left(\partial m_{2} / \partial m_{1}\right)_{\mu 0}$ in the limit of $m_{1}$ 

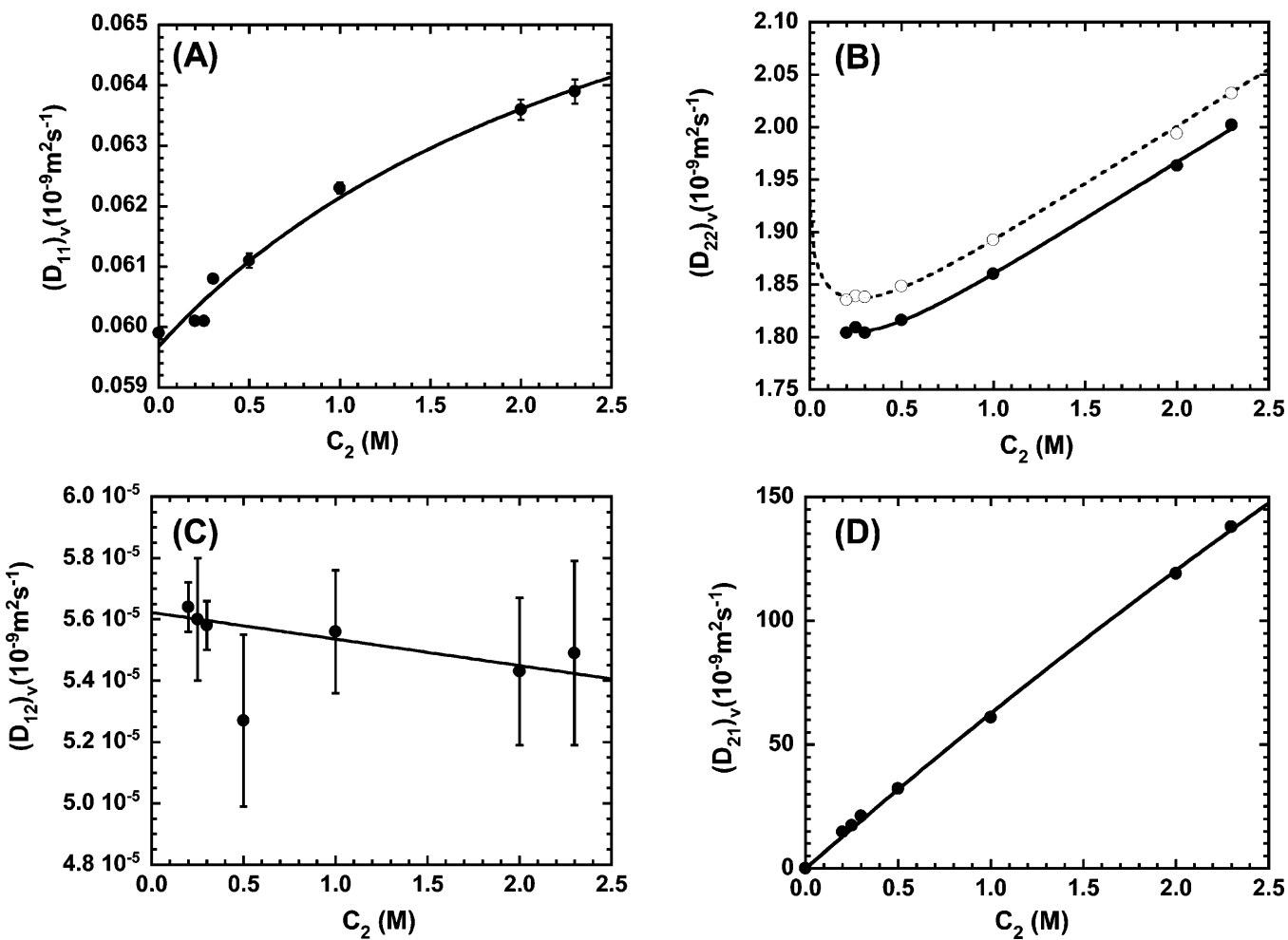

Figure 1. Volume-fixed diffusion coefficients as functions of $\mathrm{KCl}$ concentration, $C_{2}$, for the $\mathrm{PEG}-\mathrm{KCl}-\mathrm{H}_{2} \mathrm{O}$ system at $C_{1}=0.25 \mathrm{mM}$ and $25{ }^{\circ} \mathrm{C}$ : (a) $\left(D_{11}\right)_{\mathrm{V}}$; (b) $\left(D_{22}\right)_{\mathrm{V}}$; (c) $\left(D_{12}\right)_{\mathrm{V}}$; (d) $\left(D_{21}\right)_{\mathrm{V}}$. The solid curves are fits through the ternary experimental points. The dashed curve (data points omitted) is a fit through the binary $\mathrm{KCl}$ diffusion coefficients taken from ref 33 . The open circles are our measured binary values.

approaching zero. In this limit, we also obtain: $\left(\partial m_{2} / \partial m_{1}\right)_{\mu 0}=$ $\left(\partial m_{2} / \partial m_{1}\right)_{\mu 2}$ according to the Gibbs-Duhem equation.

\section{Results}

In this section, we describe our results for ternary diffusion coefficients for the PEG (1)- $\mathrm{KCl}(2)$-water (0) system at $25{ }^{\circ} \mathrm{C}$. In Table 1, we report experimental parameters related to the diffusion experiments for the ternary PEG (1) $-\mathrm{KCl}(2)-$ water (0) system at seven mean $\mathrm{KCl}$ concentrations $\bar{C}_{2}=0.20$, $0.25,0.30,0.50,1.00,2.00$, and $2.30 \mathrm{M}$ and the same mean PEG concentration of $\bar{C}_{1}=0.25 \mathrm{mM}$. We observe that this concentration falls inside the dilute-solution regime and is lower than the PEG concentrations normally employed for the precipitation of biomacromolecules. ${ }^{17,18}$ The volume-frame diffusion coefficients $\left(D_{i j}\right)_{\mathrm{V}}$ and the corresponding eigenvalues, $\lambda_{1}$ and $\lambda_{2}$, were obtained by applying the method of the nonlinear least-square as described ref 32. In Table 1, we also report the partial molar volumes: $\overline{\mathrm{V}}_{1}, \overline{\mathrm{V}}_{2}$, and $\overline{\mathrm{V}}_{0}$, obtained from density measurements. The $\overline{\mathrm{V}}_{i}$ 's were used to calculate the solvent-frame diffusion coefficients $\left(D_{i j}\right)_{0}$ from the $\left(D_{i j}\right)_{\mathrm{V}}$ 's using eq $2 \mathrm{a}-\mathrm{d}$. The chemical potential derivative, $\mu_{11}^{(m)}$, was calculated using: $\mu_{11}^{(m)} / R T=1 / m_{1}$. On the other hand, the chemical-potential derivative, $\mu_{22}^{(m)}$, was calculated from the available activity coefficient data of the binary $\mathrm{KCl}$-water system taken at the same $m_{2}$ values. ${ }^{33}$ Since the experimental PEG concentration is low, the preferential interaction coefficient, $\Gamma_{12}$, is calculated using eq 9 with the approximation that $\Gamma_{12}=-\mu_{12}^{(m)} / \mu_{22}^{(m)} \cdot{ }^{13}$ The calculated values of $\Gamma_{12}$ are reported as a function of $\mathrm{KCl}$ concentration. Experimental details related to individual ternary diffusion experiments and binary experiments on the $\mathrm{KCl}$-water system are available as Supporting Information.

In Figure $1 \mathrm{a}-\mathrm{d}$, we illustrate the behavior of the four $\left(D_{i j}\right)_{\mathrm{V}}$ as a function of salt concentration. In Figure 1a, we show that $\left(D_{11}\right)_{\mathrm{V}}$ increases with $C_{2}$. This implies that PEG diffusion is

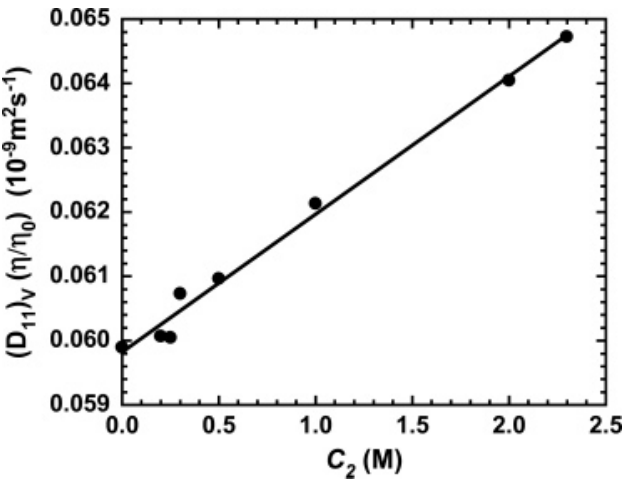

Figure 2. Viscosity-corrected $\left(D_{11}\right)_{\mathrm{v}}\left(\eta / \eta_{0}\right)$ as a function of salt concentration, $C_{2}$, for the PEG- $\mathrm{KCl}-\mathrm{H}_{2} \mathrm{O}$ system. The solid line is a linear fit through the experimental points. The viscosity coefficients of the binary salt-water systems were taken from ref 37 .

enhanced in the presence of salt. At low concentration of neutral macromolecules, we expect that $\left(D_{11}\right)_{\mathrm{V}} \approx D_{\mathrm{m}},{ }^{34,35}$ where $D_{\mathrm{m}}$ is the infinite dilution tracer diffusion coefficient of the macromolecule. Indeed our experimental value for the binary PEGwater system at $C_{1}=0.25 \mathrm{mM}$ is $\left(D_{11}\right)_{\mathrm{v}}=0.05990 \times 10^{-9}$ $\mathrm{m}^{2} \mathrm{~s}^{-1}$. This value is $4 \%$ higher than the corresponding tracer diffusion coefficient $\left(D_{\mathrm{m}}=0.0574 \times 10^{-9} \mathrm{~m}^{2} \mathrm{~s}^{-1}\right) .{ }^{36}$ According to the Stokes-Einstein equation, ${ }^{35}$ the isothermal dependence of $D_{\mathrm{m}}$ on salt concentration can be attributed to changes in salt water viscosity and macromolecule hydrodynamic radius. To remove the effect of viscosity, we consider the product: $\left(D_{11}\right)_{\mathrm{V}^{-}}$ $\left(\eta / \eta_{0}\right)$, where $\eta$ is the viscosity of the corresponding binary $\mathrm{KCl}-$ water system ${ }^{37}$ and $\eta_{0}$ is the viscosity of water. In Figure 2 , we plot $\left(D_{11}\right)_{\mathrm{V}}\left(\eta / \eta_{0}\right)$ as a function of $C_{2}$. This plot shows that $\left(D_{11}\right)_{\mathrm{V}}\left(\eta / \eta_{0}\right)$ increases linearly with salt concentration. At the highest experimental salt concentration, its value is $8.2 \%$ higher than that at $C_{2}=0$. The observed behavior is consistent with a corresponding decrease of the PEG hydrodynamic radius. 
This polymer collapse can be related to a decrease of solvent quality in the presence of salt, consistent with the known capability of salts to enhance liquid-liquid-phase separation of PEG aqueous solutions. ${ }^{38}$ As we shall discuss later, the dependence of the preferential interaction coefficient on salt concentration is also consistent with the observed behavior of $\left(D_{11}\right)_{\mathrm{V}}\left(\eta / \eta_{0}\right)$.

In Figure 1b, we show the behavior of the salt main diffusion coefficient $\left(D_{22}\right)_{\mathrm{V}}$ (solid circles). In the same figure, we also include the corresponding binary diffusion coefficients (open circles) and the available literature data (dashed curve). ${ }^{33} \mathrm{We}$ can see that our binary measurements are in very good agreement with literature data. Moreover, our ternary data show the same behavior as the corresponding binary curve. This is strong evidence that using binary thermodynamic data to calculate ternary $\mu_{22}^{(m)}$ values is a very good approximation. ${ }^{6}$ We can relate our ternary data to the corresponding binary data by $\left(D_{22}\right)_{\mathrm{V}}=(1-k \phi)\left(D_{2}\right)_{\mathrm{V}}$, where $\phi=C_{1} \bar{V}_{1}$ is the polymer volume fraction and $k$ is a constant factor that characterizes a small obstruction effect of the PEG macromolecules on the motion of the small salt ions. It has been theoretically shown that $k=1.5$ for small solutes in the presence of hard spheres. ${ }^{39}$ For aqueous $\mathrm{KCl}$ in the presence of PEG, we obtain $k=4.0 \pm$ 0.2 by fitting the experimental $\left(D_{22}\right)_{\mathrm{V}}$ using the available expression of $\left(D_{2}\right)_{\mathrm{V}}$ and our experimental PEG volume fraction, $\phi=0.0042$. For comparison, we have also calculated $k$ for aqueous $\mathrm{KCl}$ in the presence of lysozyme (at $\phi=0.0061){ }^{6}$ Here, we obtain $k=1.8 \pm 0.1$, which is close to the hard-sphere case. We therefore conclude that the obstruction effect of PEG on the salt ions is 2-fold higher than that of lysozyme. This result is consistent with polymer coils being significantly more expanded than globular compact proteins.

In Figure 1c,d, we show the behavior of the two crossdiffusion coefficients $\left(D_{12}\right)_{\mathrm{V}}$ and $\left(D_{21}\right)_{\mathrm{V}}$. We can see that $\left(D_{12}\right)_{\mathrm{V}}$ is positive and approximately independent of $C_{2}$ within the experimental error. A positive value of $\left(D_{12}\right)_{\mathrm{V}}$ implies that at uniform PEG concentration the macromolecules diffuse from high to low salt concentration. In Figure 1d, we show that $\left(D_{21}\right)_{\mathrm{V}}$ is positive and increases with $C_{2}$. Note that the value of $\left(D_{21}\right)_{\mathrm{V}}$ must approach zero at $C_{2}=0$. This is because the salt flux becomes zero, independent of PEG concentration gradient. A positive value of $\left(D_{21}\right)_{\mathrm{V}}$ implies that salt diffuses from high to low PEG concentration in the presence of uniform salt concentration. The behavior of both cross-diffusion coefficients is consistent with the presence of net repulsive interactions between $\mathrm{PEG}$ and $\mathrm{KCl}$ in water. We will further examine the crossdiffusion coefficients in the Discussion section.

To validate the accuracy of the $\Gamma_{12}$ values reported in Table 1 , we have also used the isopiestic method for the determination of the preferential interaction coefficients. Typically, the isopiestic method has been employed for the determination of activity coefficients of salt-water mixtures. ${ }^{23}$ To our knowledge, this is the first time that this method is used for the determination of preferential interaction coefficients of macromolecules. In Figure 3, measurements of $\left(\Delta m_{2} / \Delta m_{1}\right)_{\mu_{0}=\mu_{0}^{\prime}}$ are shown for two chosen $\mathrm{KCl}$ concentrations: $C_{2}=0.25$ and $1.0 \mathrm{M}$. These two sets of data were fitted using the linear weighed least-square method. The corresponding intercepts are the values of $-\Gamma_{12}$ (see Materials and Methods). We obtain: $-\Gamma_{12}=5.2 \pm 0.4$ at $C_{2}=0.25 \mathrm{M}$ and $-\Gamma_{12}=16.6 \pm 0.7$ at $C_{2}=1.0 \mathrm{M}$. Here the reported errors are standard deviations. The corresponding values extracted from the ternary diffusion coefficients are: $-\Gamma_{12}=$ $5.7 \pm 0.2$ and $-\Gamma_{12}=17.4 \pm 0.2$ (see Table 1 ). We therefore

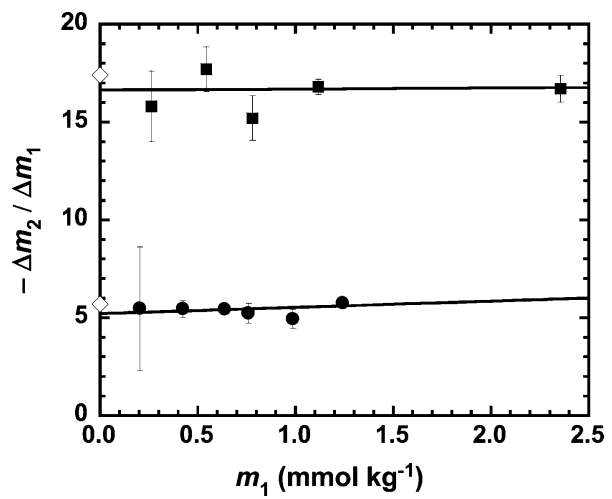

Figure 3. Molality ratios $-\Delta m_{2} / \Delta m_{1}$ as a function of PEG molality, $m_{1}$, obtained at $C_{2}=0.25 \mathrm{M}$ (solid circles) and $C_{2}=1 \mathrm{M}$ (solid squares) using the isopiestic method. The solid lines are weighted linear fits through the data. The preferential interaction coefficients obtained using ternary diffusion at $C_{2}=0.25 \mathrm{M}$ and $C_{2}=1 \mathrm{M}$ (open diamonds) are included for comparison.

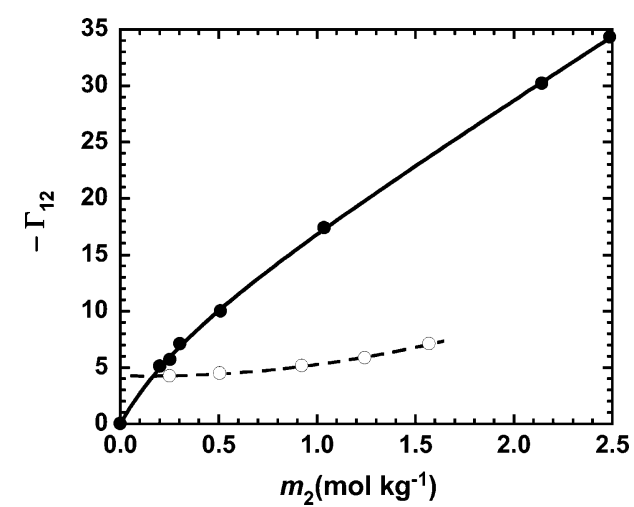

Figure 4. Preferential interaction coefficients obtained using ternary diffusion reported as $-\Gamma_{12}$ as a function of salt molality, $m_{2}$, for the $\mathrm{PEG}-\mathrm{KCl}-\mathrm{H}_{2} \mathrm{O}$ (solid circles) and the lysozyme $-\mathrm{KCl}-\mathrm{H}_{2} \mathrm{O}$ (open circles) systems. The curves are fits through the data.

conclude that diffusion and isopiestic measurements are in very good agreement within the experimental error.

\section{Discussion}

In this section, we will first discuss the preferential interaction coefficients reported in Table 1 and compare these values with those previously obtained for the lysozyme- $\mathrm{KCl}$-water system at $\mathrm{pH} 4.5 .^{6}$ We will then examine the more complex behavior of the cross-diffusion coefficients in relation to the system thermodynamic behavior.

Preferential Interaction Coefficients. In Figure 4, we plot $-\Gamma_{12}$ as a function of salt molality $m_{2}$ for both PEG (solid circles) and lysozyme (open circles) cases. The first important difference between the PEG and lysozyme case occurs at $m_{2}=$ 0 . Since lysozyme at $\mathrm{pH} 4.5$ is positively charged, we have $-\Gamma_{12}$ $=z_{\mathrm{P}} / 2$ at $m_{2}=0$ (with $z_{\mathrm{p}} m_{1} \ll m_{2}$ ), where $z_{\mathrm{P}}$ is the protein charge. ${ }^{12,40}$ For lysozyme, we have previously reported $z_{\mathrm{p}}=8$ \pm 1.6 This value corresponds to the protein effective charge and is $\approx 30 \%$ lower than the net titration charge, ${ }^{41}$ due to counterion binding. ${ }^{6}$ On the other hand, since PEG is a neutral macromolecule, we have $-\Gamma_{12}=0$ at $m_{2}=0$ (see Figure 3).

We now discuss the dependence of $-\Gamma_{12}$ on salt concentration. In both cases, $-\Gamma_{12}$ increases with $m_{2}$, consistent with $\mathrm{PEG}-\mathrm{KCl}$ net repulsive interactions. However, the increase observed in the PEG case is significantly larger. To provide a physical interpretation on the difference in slope between the two macromolecules, we can use the two-domain model. ${ }^{2,40}$ 


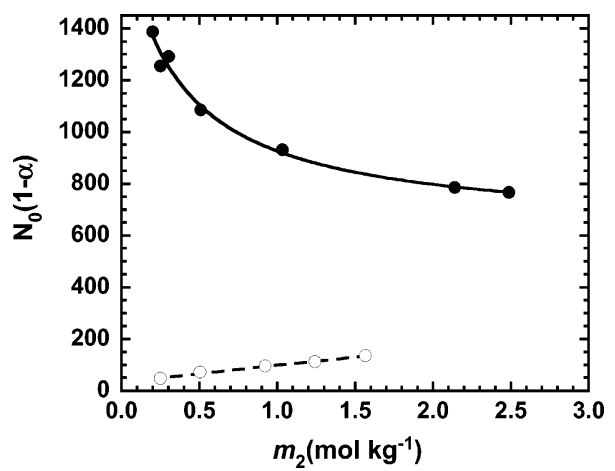

Figure 5. Water excess, $N_{0}(1-\alpha)$, a functions of $\mathrm{KCl}$ concentration, $\mathrm{C}_{2}$, for the $\mathrm{PEG}-\mathrm{KCl}-\mathrm{H}_{2} \mathrm{O}$ (solid circles) and the lysozyme- $\mathrm{KCl}-$ $\mathrm{H}_{2} \mathrm{O}$ (open circles) systems. The curves are fits through the data. The values of $N_{0}(1-\alpha)$ for the PEG case were calculated using $\left(-\Gamma_{12}\right)$ $\left(m_{0} / m_{2}\right)$. The values of $N_{0}(1-\alpha)$ for the lysozyme case were calculated using $\left(-\Gamma_{12}-z_{\mathrm{P}} / 2\right)\left(m_{0} / m_{2}\right)$ with $z_{\mathrm{P}}=8$.

According to this model, the first domain is represented by the water-osmolyte layers surrounding a macromolecule. This local domain is in chemical equilibrium with a bulk domain representing the water-osmolyte remaining solution. Since macromolecules interact with the osmolyte and water molecules in their vicinity, the concentration of osmolyte in the local domain is different from that of the unperturbed bulk domain. It can be shown $n^{13,40}$ that the slope of $-\Gamma_{12}\left(m_{2}\right)$ is $\left(N_{0} / m_{0}\right)(1-\alpha)$, where $N_{0}$ is the number of water molecules of the local domain, $\alpha$ is the osmolyte partitioning constant between the local and the bulk domains, and $m_{0}=55.51 \mathrm{~mol} \mathrm{~kg}^{-1}$. If $\alpha<1$, i.e., the slope of $-\Gamma_{12}\left(m_{2}\right)$ is positive, the salt concentration of the local domain is lower than that of the bulk domain. Hence, our results in Figure 4 show that both PEG and lysozyme are preferentially hydrated in the presence of $\mathrm{KCl}$. Moreover, we can use the $\Gamma_{12}$ values to calculate $N_{0}(1-\alpha)$. This quantity represents the excess of water molecules in the local domain. In Figure 5, we plot (see $N_{0}\left(1-\alpha\right.$ ) as a function of $C_{2}$ for PEG and lysozyme. We can see that the water excess for PEG is considerably larger than that for lysozyme, even though the molecular weight of lysozyme is only $30 \%$ lower than that of PEG. This large difference in water excess can be explained by considering the structural difference between a protein and polymer. Since lysozyme is a globular compact protein, only its surface is accessible to the surrounding fluid. On the other hand, a polymer molecule behaves like a coil with all its monomeric units accessible to the surrounding fluid and, therefore, is able to interact with the salt ions. Furthermore, Figure 5 also shows that the water excess for the PEG case significantly decreases as salt concentration increases. This can be related to the deformability of PEG coils. This observed decrease in water excess is also consistent with a disruption of structured water surrounding the PEG macromolecules induced by the presence of salt ions. As $C_{2}$ increases, the polymer coil collapses on itself due to $\mathrm{PEG}-\mathrm{KCl}$ net repulsive interactions. Clearly, this result is consistent with the salt-dependence of $\left(D_{11}\right)_{\mathrm{V}}\left(\eta / \eta_{0}\right)$ shown in Figure 2.

Examination of Cross-Diffusion Coefficients. We will now discuss the behavior of the cross-diffusion coefficients in relation to the main diffusion coefficients and the preferential interaction coefficients. We will consider the solvent-frame diffusion coefficients, due to their direct relation to chemical potential derivatives (see eq $5 \mathrm{a}-\mathrm{d}$ ). The difference between the volumeframe and solvent-frame values is small and marginal for the interpretations reported below. We will specifically address the behavior of the ratios: $\left(D_{12}\right)_{0} /\left(D_{11}\right)_{0}$ and $\left(D_{21}\right)_{0} /\left(D_{22}\right)_{0}$. This
TABLE 2: Comparison between $\left(D_{i j}\right)_{0} /\left(D_{i i}\right)_{0}$ and $\mu_{i j}^{(c)} / \mu_{i i}^{(c)}$ Ratios

\begin{tabular}{llllllll}
\hline $\bar{C}_{2}(\mathrm{M})$ & 0.20 & 0.25 & 0.30 & 0.50 & 1.00 & 2.00 & 2.30 \\
$\left(D_{12}\right)_{0} /\left(D_{11}\right)_{0}$ & 0.0012 & 0.0011 & 0.0011 & 0.0011 & 0.0011 & 0.0011 & 0.0011 \\
$\mu_{12}^{(c)} / \mu_{11}^{(c)}$ & 0.0114 & 0.0106 & 0.0109 & 0.0094 & 0.0085 & 0.0081 & 0.0082 \\
$\left(D_{11} / D_{0} /\left(D_{22}\right)_{0}\right.$ & 8.1 & 9.8 & 11.9 & 18.0 & 33.4 & 61.7 & 70.1 \\
$\mu_{21}^{(c)} / \mu_{22}^{(c)}$ & 8.3 & 9.9 & 12.1 & 18.3 & 33.8 & 62.5 & 71.0
\end{tabular}

allows us to remove the explicit dependence of the crossdiffusion coefficients on the tracer diffusion coefficients of macromolecule and salt.

The two diffusion-coefficient ratios can be expressed in terms of the chemical potential derivatives, $\mu_{i j}^{(c)}$, using eq $5 \mathrm{a}-\mathrm{d}$ :

$$
\begin{aligned}
& \frac{\left(D_{12}\right)_{0}}{\left(D_{11}\right)_{0}}=\frac{\mu_{12}^{(c)}+\left[\left(L_{12}\right)_{0} /\left(L_{11}\right)_{0}\right] \mu_{22}^{(c)}}{\mu_{11}^{(c)}+\left[\left(L_{12}\right)_{0} /\left(L_{11}\right)_{0}\right] \mu_{21}^{(c)}} \\
& \frac{\left(D_{21}\right)_{0}}{\left(D_{22}\right)_{0}}=\frac{\mu_{21}^{(c)}+\left[\left(L_{12}\right)_{0} /\left(L_{22}\right)_{0}\right] \mu_{11}^{(c)}}{\mu_{22}^{(c)}+\left[\left(L_{12}\right)_{0} /\left(L_{22}\right)_{0}\right] \mu_{12}^{(c)}}
\end{aligned}
$$

If $\left(L_{12}\right)_{0}=0$, the ratios: $\left(D_{12}\right)_{0} /\left(D_{11}\right)_{0}$ and $\left(D_{21}\right)_{0} /\left(D_{22}\right)_{0}$ become equal to the thermodynamic ratios: $\mu_{12}^{(c)} / \mu_{11}^{(c)}$ and $\mu_{21}^{(c)} / \mu_{22}^{(c)}$, respectively. Indeed the Nernst-Hartley equations, ${ }^{15}$ which are valid at infinite dilution, predict $\left(L_{12}\right)_{0}<0$ for charged macromolecules, and $\left(L_{12}\right)_{0} \rightarrow 0$ as the charge on the macromolecule becomes zero. This limiting case applies to PEG. We will now examine if Onsager cross-diffusion coefficients can be indeed neglected for neutral macromolecules by numerically comparing our diffusion and the thermodynamic ratios. The corresponding values are reported in Table 2 . We can see that $\left(D_{12}\right)_{0} /\left(D_{11}\right)_{0}$ is 1 order of magnitude lower than $\mu_{12}^{(c)} / \mu_{11}^{(c)}$ within our salt concentration range. Although we have assumed $\mu_{11}^{(c)}=$ $R T / C_{1}$, this approximation is not expected to significantly affect the observed large discrepancy. We therefore conclude that the assumption $\left(L_{12}\right)_{0}=0$ in eq 11 a cannot be applied for the interpretation of $\left(D_{12}\right)_{0} /\left(D_{11}\right)_{0}$. On the other hand, we can see in Table 2 that the discrepancy between $\left(D_{21}\right)_{0} /\left(D_{22}\right)_{0}$ and $\mu_{21}^{(c)} /$ $\mu_{22}^{(c)}$ is only $1-2 \%$. Thus $\left(L_{12}\right)_{0}$ does not play an important role in eq $11 \mathrm{~b}$, and $\left(D_{21}\right)_{0} /\left(D_{22}\right)_{0}$ is essentially a thermodynamic quantity as shown by eq 10 . The nearly equivalence between $\left(D_{21}\right)_{0} /\left(D_{22}\right)_{0}$ and $\mu_{21}^{(c)} / \mu_{22}^{(c)}$ can be understood by considering the diffusion experiment for which an initial sharp boundary exists between two solutions having same salt concentration and different macromolecule concentrations. Since macromolecules diffuse relatively slowly, the fast-diffusing salt component (and the solvent) will reach a quasi-equilibrium condition, $\left(J_{2}\right)_{0} \approx$ 0 , in the presence of a slowly dissipating concentration gradient of macromolecules. According to eq $1 \mathrm{~b}$, this implies that $\left(D_{21}\right)_{0} \nabla C_{1} \approx-\left(D_{22}\right)_{0} \nabla C_{2}$ or $\left(D_{21}\right)_{0} /\left(D_{22}\right)_{0} \approx-\left(\partial C_{2} / \partial C_{1}\right) \hat{\mu}_{2}=$ $\mu_{21}^{(c)} / \mu_{22}^{(c)}$. Clearly, this interpretation cannot be extended to the opposite diffusion experiment with the two solutions having same macromolecule concentration and different salt concentration. This is because a salt concentration gradient will dissipate relatively fast. Thus $\left(D_{12}\right)_{0} /\left(D_{11}\right)_{0}$ is expected to significantly depend on kinetic parameters. For ternary systems, with two solutes of different size, we can therefore conclude that only the salt diffusion ratio, $\left(D_{21}\right)_{0} /\left(D_{22}\right)_{0}$, can be interpreted merely using thermodynamic quantities. On the other hand, the contribution of $\left(L_{12}\right)_{0}$ cannot be neglected for the interpretation of the macromolecule diffusion ratio, $\left(D_{12}\right)_{0} /\left(D_{11}\right)_{0}$, even for neutral macromolecules.

We now compare our results on $\left(D_{12}\right)_{0} /\left(D_{11}\right)_{0}$ and $\left(D_{21}\right)_{0} /$ $\left(D_{22}\right)_{0}$ for the PEG case with those previously obtained for the 


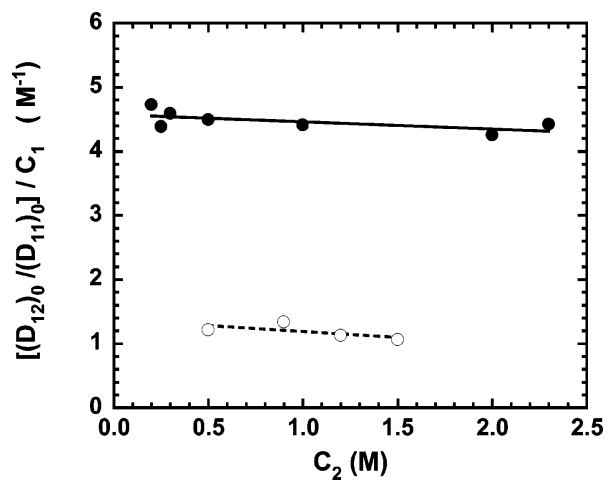

Figure 6. The ratio $\left[\left(D_{12}\right)_{0} /\left(D_{11}\right)_{0}\right] / C_{1}$ as a function of salt concentration, $\mathrm{C}_{2}$, for the $\mathrm{PEG}-\mathrm{KCl}-\mathrm{H}_{2} \mathrm{O}$ (solid circles) and the lysozyme- $\mathrm{KCl}-$ $\mathrm{H}_{2} \mathrm{O}$ (open circles) systems. The curves are linear fits through the data.

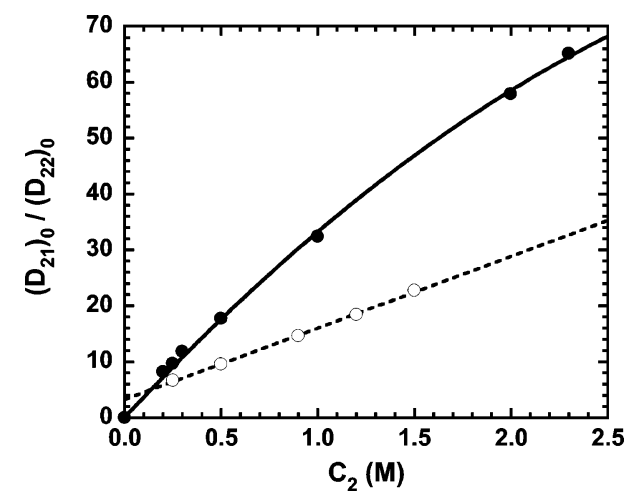

Figure 7. The ratio $\left(D_{21}\right)_{0} /\left(D_{22}\right)_{0}$ as a function of salt concentration, $\mathrm{C}_{2}$, for the PEG- $\mathrm{KCl}-\mathrm{H}_{2} \mathrm{O}$ (solid circles) and the lysozyme- $\mathrm{KCl}-$ $\mathrm{H}_{2} \mathrm{O}$ (open circles) systems. The curves are linear fits through the data.

lysozyme case. ${ }^{6}$ We note that, in the limit of $C_{1} \rightarrow 0$, the denominator of eq 11a approaches $\mu_{11}^{(c)}=1 / C_{1}$ and $\left(D_{12}\right)_{0} /\left(D_{11}\right)_{0}$ becomes directly proportional to $C_{1}$. To remove this expected $C_{1}$ dependence, the comparison between these two macromolecules is performed by examining $\left[\left(D_{12}\right)_{0} /\left(D_{11}\right)_{0}\right] / C_{1}$ as a function of $C_{2}$. In Figure 6, we can see that the values corresponding to the PEG case are about 4-fold larger than those related to the lysozyme case. Although the ratio $\left(D_{12}\right)_{0} /\left(D_{11}\right)_{0}$ significantly depends on both thermodynamic and transport parameters, the difference in $\left[\left(D_{12}\right)_{0} /\left(D_{11}\right)_{0}\right] / C_{1}$ between the two macromolecules implies that PEG affinity for water and, consequently, its tendency to migrate toward lower salt concentration are higher than those of the lysozyme. This is consistent with our preferential hydration results in Figure 5.

In Figure 7 , we show the $\left(D_{21}\right)_{0} /\left(D_{22}\right)_{0}$ values for the PEG and lysozyme cases as a function of $C_{2}$. The corresponding curves increase with salt concentrations and display a behavior similar to that shown in Figure 4 for $-\Gamma_{12}$. This is consistent with eq 10 , which shows that the values of the $\left(D_{21}\right)_{0} /\left(D_{22}\right)_{0}$ slopes are systematically higher than the corresponding values of $-\Gamma_{12}$, due to the positive contribution of $C_{2} \overline{\mathrm{V}}_{1}$.

\section{Conclusions}

We have reported ternary diffusion coefficients for the PEG$\mathrm{KCl}$-water system. First, the experimental comparison between diffusion and isopiestic measurements has allowed us to demonstrate that multicomponent diffusion coefficients can be used to determine preferential interaction coefficients. This result is not only important for the examination of the accuracy of the thermodynamic data obtained using ternary diffusion but it also represents a significant contribution to the validation of three postulates of nonequilibrium thermodynamics applied to diffusion processes involving macromolecules. Second, we have observed a large preferential hydration of the PEG coils in the presence of $\mathrm{KCl}$. This hydration was found to be about 1 order of magnitude larger than that observed for lysozyme, a protein of similar molecular weight. Third, the diffusion coefficient ratios: $\left(D_{12}\right)_{0} /\left(D_{11}\right)_{0}$ and $\left(D_{21}\right)_{0} /\left(D_{22}\right)_{0}$, which describe the magnitude of cross-diffusion coefficients, are larger for the $\mathrm{PEG}-\mathrm{KCl}-$ water system than those for the lysozyme- $\mathrm{KCl}-$ water system at the same solute concentrations. This difference can be related to the corresponding difference in preferential hydration. We have also established that only the diffusion ratio $\left(D_{21}\right)_{0} /\left(D_{22}\right)_{0}$ is essentially a thermodynamic quantity. The other diffusion ratios strongly depend on the Onsager cross-diffusion coefficient, $\left(L_{12}\right)_{0}$, even for neutral macromolecules.

Acknowledgment. This work was supported by the ACS Petroleum Research Fund (47244-G4) and TCU Research and Creative Activity Funds.

Supporting Information Available: Experimental details related to individual ternary diffusion experiments and binary experiments on the $\mathrm{KCl}$-water system. This material is available free of charge via the Internet at http://pubs.acs.org.

\section{References and Notes} 7367.

(1) Timasheff, S. N. Proc. Natl. Acad. Sci. U.S.A. 1998, 95, 7363-

(2) Parsegian, V. A.; Rand, R. P.; Rau. D. C. Proc. Natl. Acad. Sci. U.S.A. 2000, 97, 3987-3992. 9726.

(3) Timasheff, S. N. Proc. Natl. Acad. Sci. U.S.A. 2002, 99, 9721-

(4) Schellman, J. A. Q. Rev. Biophys. 2005, 38, 351-361.

(5) Wyman, J., Jr. Adv. Protein Chem. 1964, 19, 223-286.

(6) Annunziata, O.; Paduano, L.; Pearlstein, A. J.; Miller, D. G.; Albright, J. G. J. Phys. Chem. B 2006, 110, 1405-1415.

(7) Arakawa, T.; Bhat, R.; Timasheff, S. N. Biochemistry 1990, 29, $1914-1923$

(8) Courtneay, E. S.; Capp, M. W.; Anderson, C. F.; Record, M. T., Jr. Biochemistry 2000, 39, 4455-4471.

(9) Leaist, D. G. J. Phys. Chem. 1989, 93, 474-479.

(10) Albright, J. G.; Annunziata, O.; Miller, D. G.; Paduano, L.; Pearlstein, A. J. J. Am. Chem. Soc. 1999, 121, 3256-3266.

(11) Annunziata, O.; Paduano, L.; Pearlstein, A. J.; Miller, D. G.; Albright, J. G. J. Am. Chem. Soc. 2000, 122, 5916-5928.

(12) Annunziata, O.; Paduano, L.; Albright, J. G. J. Phys. Chem. B 2006, $110,16139-16147$

(13) Annunziata, O.; Paduano, L.; Albright, J. G. J. Phys. Chem. B 2007, $111,10591-10598$.

(14) We use the subscript "1" for the macromolecule and the subscript " 2 " for the osmolyte, consistent with the literature on multicomponent diffusion. However, the subscript "2" for the macromolecule and the subscript " 3 " for the osmolyte are extensively used in relation to the thermodynamic description of protein mixtures.

(15) Gosting, L. J. Adv. Protein Chem. 1956, 11, 429-554.

(16) Paduano, L.; Annunziata, O.; Pearlstein, A. J.; Miller, D. G.; Albright, J. G. J. Cryst. Growth 2001, 232, 273-284.

(17) Albertsson, P. A. Partition of Cell Particles and Macromolecules; Wiley: New York, 1986.

(18) McPherson, A. Crystallization of Biological Macromolecules; Cold Spring Harbor: New York, 1998.

(19) Edlund, U.; Albertsson, A. C. Adv. Polym. Sci. 2002, 157, 67112.

(20) Vergara, A.; Annunziata, O.; Paduano, L.; Miller, D. G.; Albright, J. G.; Sartorio, R. J. Phys. Chem. B 2004, 108, 2764-2772.

(21) Capuano, F.; Vergara, A.; Paduano, L.; Annunziata, O.; Sartorio, R. J. Phys. Chem. B 2003, 107, 12363-12369.

(22) Fitts, D. D. Nonequilibrium Thermodynamics: A Phenomenological Theory of Irreversible Processes in FLuid Systems; McGraw-Hill: New York, 1962.

(23) Albright, J. G.; Rizzo, P.; Rard, J. A. J. Chem. Thermodyn. 1998, $30,327-352$.

(24) Kirkwood, J. G.; Baldwin, R. L.; Dunlop, P. J.; Gosting, L. J.; Kegeles, G. J. Chem. Phys. 1960, 33, 1505-1513. 
(25) Miller, D. G.; Vitagliano, V.; Sartorio, R. J. Phys. Chem. 1986, 90, 1509-1519.

(26) Dunlop, P. J.; Gosting, L. J. J. Phys. Chem. 1959, 63, 86-93.

(27) Woolf, L. A.; Miller, D. G.; Gosting, L. J. J. Am. Chem. Soc. 1962 $84,317-331$

(28) Onsager, L. Phys. Rev. 1931, 38, 2265-2279.

(29) Miller, D. G. J. Phys. Chem. 1959, 63, 570-578.

(30) Vergara, A.; Paduano, L.; Sartorio, R.; Vitagliano, V. J. Phys. Chem. B 1999, 103, 8732-8738.

(31) Miller, D. G.; Albright, J. G. In Measurement of the Transport Properties of Fluids: Experimental Thermodynamics; Wakeham, W. A., Nagashima, A., Sengers, J. V., Eds.; Blackwell Scientific Publications: Oxford, 1991; Vol. 3, pp 272-294.

(32) Miller, D. G. J. Phys. Chem. 1988, 92, 4222-4226.

(33) Rard, A. J.; Miller, D. G. J. Chem. Eng. Data 1980, 25, 211-215.
(34) Annunziata, O.; Buzatu, D.; Albright, J. G. Langmuir 2005, 21, 12085-12089.

(35) Tanford, C. Physical Chemistry of Macromolecules; Wiley: New York, 1961

(36) Vergara, A.; Paduano, L.; Sartorio, R. J. Polym. Sci., Part B: Polym. Phys. 2002, 40, 43-51.

(37) Zhang, H.-L.; Han, S.-J. J. Chem. Eng. Data 1996, 41, 516-520.

(38) Taboada, M. E.; Galleguillos, H. R.; Graber, T. A.; Bolado, S. J. Chem. Eng. Data 2005, 41, 264-269.

(39) Sridharan, S.; Cukier, R. I. J. Phys. Chem. 1984, 88, 1237-1242.

(40) Record, M. T., Jr.; Anderson, C. F. Biophys. J. 1995, 68, 786794.

(41) Tanford, C.; Wagner, M. L. J. Am. Chem. Soc. 1954, 76, 33313336. 